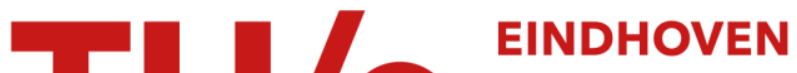 \\ UNIVERSITY OF \\ TECHNOLOGY
}

\section{Continuous time vertex-reinforced jump processes}

Citation for published version (APA):

Davis, B., \& Volkov, S. E. (2001). Continuous time vertex-reinforced jump processes. (Report Eurandom; Vol. 2001003). Technische Universiteit Eindhoven.

Document status and date:

Published: 01/01/2001

\section{Document Version:}

Publisher's PDF, also known as Version of Record (includes final page, issue and volume numbers)

\section{Please check the document version of this publication:}

- A submitted manuscript is the version of the article upon submission and before peer-review. There can be important differences between the submitted version and the official published version of record. People interested in the research are advised to contact the author for the final version of the publication, or visit the $\mathrm{DOI}$ to the publisher's website.

- The final author version and the galley proof are versions of the publication after peer review.

- The final published version features the final layout of the paper including the volume, issue and page numbers.

Link to publication

\section{General rights}

Copyright and moral rights for the publications made accessible in the public portal are retained by the authors and/or other copyright owners and it is a condition of accessing publications that users recognise and abide by the legal requirements associated with these rights.

- Users may download and print one copy of any publication from the public portal for the purpose of private study or research.

- You may not further distribute the material or use it for any profit-making activity or commercial gain

- You may freely distribute the URL identifying the publication in the public portal.

If the publication is distributed under the terms of Article 25fa of the Dutch Copyright Act, indicated by the "Taverne" license above, please follow below link for the End User Agreement:

www.tue.nl/taverne

Take down policy

If you believe that this document breaches copyright please contact us at:

openaccess@tue.nl

providing details and we will investigate your claim. 


\title{
Continuous time vertex-reinforced jump processes
}

\author{
Burgess Davis*and Stanislav Volkov ${ }^{\dagger \ddagger}$
}

December 1, 2000

\begin{abstract}
We study the continuous time integer valued process $X_{t}, t \geq 0$, which jumps to each of its two nearest neighbors at the rate of one plus the total time the process has previously spent at that neighbor. We show that the proportion of the time before $t$ which this process spends at integers $j$ converges to positive random variables $V_{j}$, which sum to one, and whose joint distribution is explicitly described. We also show $\lim _{t \rightarrow \infty} \max _{0 \leq s \leq t} X_{s} / \log t=2.768 \ldots$
\end{abstract}

\section{Introduction}

This paper introduces and studies a continuous time right-continuous integer valued stochastic process which jumps only to nearest neighbors. We call this process, which was conceived by W. Werner, a vertex-reinforced jump

\footnotetext{
${ }^{*}$ Department of Statistics, Purdue University, West Lafayette, IN 47907, USA. E-mail: bdavis@stat.purdue.edu

${ }^{\dagger}$ Department of Mathematics, University of Bristol, Bristol, BS81TW, UK. E-mail: S.Volkov@bristol.ac.uk

$\ddagger$ Most of the work was done when S.V. was working at Fields Institute, Toronoto, Canada, and EURANDOM, Eindhoven, The Netherlands, and also while visiting Purdue University.
} 
process (VRJP) and for now designate it by $X_{t}, t \geq 0$. Given $\left\{X_{s}, s \leq\right.$ $\left.t, X_{t}=j\right\}$ and putting $A=1+\int_{0}^{t} I\left(X_{s}=j-1\right) d s$ and $B=1+\int_{0}^{s} I\left(X_{s}=\right.$ $j+1) d s$, the probability of a jump to $j-1(j+1)$ at a time in $(t, t+h]$ equals $A h+o(h)$ (respectively $B h+o(h)$ ), where both $o(h)$ depend only on $A$ and $B$. Thus the time elapsed after $t$ until the first jump from $j$ has an exponential distribution with rate $A+B$, and the probability the jump is to $j-1$ is $A /(A+B)$. This determines VRJP in the sense that the generator determines a Markov process, even though a VRJP is not a Markov process, and as with Markov processes an initial distribution needs to be specified to complete its description. It is easy to construct VRJP, started, say, at 0 from a sequence of i.i.d. exponential random variables of parameter 1 . Other graphs may be considered, but in this paper we will stick to the integers. We note that the first use of exponential variables in connection with (discrete time) reinforced processes was made by Herman Rubin to couple a generalized Pólya urn with a pure birth process (see Davis [3] and Sellke [8]).

Of the discrete time reinforced random walks studied in the literature, the two that seem most fundamental are the bond-reinforced random walk first studied by Coppersmith and Diaconis in [2], the paper which originated the subject, and the vertex-reinforced random walk first studied by Pemantle in [6], and later by Pemantle and Volkov [7] and Volkov [10].

The Coppersmith-Diaconis walk on the integers starts with weight one on all the "bonds" $(i, i+1)$, and between times $n$ and $n+1$ jumps to one of the two nearest neighbors with probabilities summing to 1 and proportional to the weights of the bonds connecting the current state with these neighbors. Coppersmith and Diaconis observed that these walks could be realized as coupled Pólya urns. This approach proves almost sure recurrence on $\mathbf{Z}^{1}$ (see Davis [3]), which here and elsewhere in this paper will mean that every integer is almost surely visited at arbitrarily large times. Later, in a series of intricate papers, a remarkably complete description of the limit- 
ing behavior of this and many related bond-reinforced walks was provided by Tóth (see [9]). Scaled properly (not $\sqrt{n}$, in the Coppersmith-Diaconis case) they converge to various previously unknown processes, some of them quite wild. Pemantle's vertex-reinforced random walk on the integers is the vertex-reinforced analog of the walk just described. Each integer initially has weight one, and this weight is augmented by one each time it is visited. This process jumps to one of its two nearest neighbors between times $n$ and $n+1$, the relative weights of the neighbors giving the probabilities of the jumps. Not only is this walk not recurrent, but It was proved in Pemantle and Volkov [7] that it eventually gets stuck on a finite set of points, and with a positive probability in exactly five states! This paragraph only scratches the surface of the subject of discrete time reinforced walks. See Davis [4], Pemantle and Volkov [7], and Tóth [9] for more, including references to papers in biology and learning theory which use discrete time reinforced walks as models, and a discussion of some processes which are limits of reinforced walks which arose in other areas of probability. Both the walks described above, and VRJPs, are close in spirit to Pólya urns, although only for the Coppersmith-Diaconis walk is the connection explicit. Reinforced Brownian motions have also been studied. See [1] for references.

This paper began as an attempt to decide whether VRJP on the integers is recurrent. It is fairly easy to show that it does not get stuck in a finite number of states, but to show recurrence is a different matter. In the following two theorems $X_{t}, t \geq 0$, will stand for VRJP on the integers started at 0 . We omit the qualification a.s. when it clearly must hold.

Theorem 1.1. The limits $V_{i}:=\lim _{t \rightarrow \infty} \frac{1}{t} \int_{0}^{t} I\left(X_{s}=i\right) d s$ exist for each integer $i$, and are positive and sum to 1 . There are i.i.d. random variables $U_{i}, 0<i<\infty$ or $-\infty<i<0$, each having the density $f_{\gamma}(x)$ given by

$$
\frac{\exp \left(-\frac{1}{2}\left(\sqrt{x}-\frac{1}{\sqrt{x}}\right)^{2}\right)}{\sqrt{2 \pi x^{3}}}
$$


such that if we put $W_{i}$ equal to $\prod_{k=1}^{i} U_{k}$ if $i>0$, equal to $\prod_{k=i}^{-1} U_{k}$ if $i<0$, and equal to 1 if $i=0$, then $V_{i}=W_{i} / \sum_{i=-\infty}^{\infty} W_{i}$.

Theorem 1.2 Let $\alpha=0.36 \ldots$ be the number explicitly given in equation (5.46). Then $\lim _{t \rightarrow \infty} \max _{0 \leq s \leq t} X_{s} / \log t=\alpha^{-1} \approx 2.77$.

Of course, symmetry gives the analog of Theorem 1.2 for minimum. Thus VRRW on the integers started at 0 has range approximately a centered interval, for all large $t$. Each of the two theorems just stated immediately implies that VRJPs are recurrent.

\section{Vertex-reinforced jump processes on $\{0,1\}$}

In exact analogy to the definition of $X_{t}, t \geq 0$, in the previous section, we can and do define vertex-reinforced jump processes $Y$ on any any connected locally-finite graph, with the initial weight of each vertex $v$ a positive number $a_{v}$, perhaps different from one, so that the weight of $v$ at time $t$ is here $L(t, v):=a_{v}+\int_{0}^{t} I\left(Y_{s}=v\right) d s$. We still call such a process a vertex-reinforced jump process (VRJP).

In this section, we study only VRJP on $\{0,1\}$ started at 0 , with initial weight $a$ at zero and $b$ at one, and we use $Z_{t}, t \geq 0$ to designate these processes. Where it might be ambiguous which initial weights we are dealing with on $\{0,1\}$, we will use $a, b$ as a superscript. The initial position is always 0 unless explicitly mentioned. Especially $\mathrm{P}^{a, b}$ and $\mathrm{E}^{a, b}$ refer only to VRJP started at 0 . At times we will need to consider random initial weights, and we will use a similar convention.

We now recall some classical results about discrete parameter martingales. Let $f_{1}, f_{2}, \ldots$ be a martingale with difference sequence $d_{1}=f_{1}$, $d_{i}=f_{i}-f_{i-1}, i>1$. Doob's maximal inequalities ( [5] (p. 308)) say

$$
\mathrm{E}\left(\sup _{n \geq 1}\left|f_{n}\right|\right)^{p} \leq\left(\frac{p}{p-1}\right)^{p} \sup _{n \geq 1} \mathrm{E}\left|f_{n}\right|^{p}, p>1
$$


If $\mathrm{E} f_{n}^{2}<\infty$ for each $n$, then $d_{i}, i \geq 1$, is an orthogonal series and thus $\mathrm{E}\left(f_{n+k}-f_{n}\right)^{2}=\sum_{i=n+1}^{n+k} \mathrm{E} d_{i}^{2}$. In addition, the almost sure convergence of $L^{2}$-bounded and thus $L^{1}$-bounded martingales, together with the fact that $f_{n+i}, i \geq 0$, is a martingale for each $i$, give with (2.1)

$$
\begin{aligned}
\mathrm{E} \sup _{k \geq 0}\left(f_{n+k}-f_{\infty}\right)^{2} & =\mathrm{E} \sup _{k \geq 0}\left[\left(f_{n+k}-f_{n}\right)-\lim _{k \rightarrow \infty}\left(f_{n+k}-f_{n}\right)\right]^{2} \\
& \leq \mathrm{E} 4 \sup _{k \geq 0}\left|f_{n+k}-f_{n}\right|^{2} \leq 16 \sum_{k>0} \mathrm{E} d_{n+k}^{2} \\
& =16 \lim _{k \rightarrow \infty} \mathrm{E}\left(f_{n+k}-f_{n}\right)^{2},
\end{aligned}
$$

if $\sup _{n} \mathrm{E} f_{n}^{2}<\infty$, where $f_{\infty}:=\lim _{i \rightarrow \infty} f_{i}$.

In the proof of the following lemma, and throughout the paper, we adopt the usual convention that $C, K$ etc. often stand for positive constants which may change from line to line.

Lemma 2.1 Let $f_{1}, f_{2}, \ldots, f_{n}$ be a martingale with differences $d_{1}, d_{2}, \ldots, d_{n}$ satisfying

$$
\max _{1 \leq j \leq n} \mathrm{E}\left(d_{j}^{4} \mid d_{i}, i<j\right)=\gamma<\infty,
$$

and let $\varepsilon>0$. Then there is a constant $K=K(\gamma, \varepsilon)$ such that

$$
\mathrm{P}\left(\max _{1 \leq j \leq n}\left|f_{i}\right|>\varepsilon n\right)<\frac{K}{n^{2}} .
$$

Proof: This proof, which is probably known, is a close cousin to the standard proof of complete convergence of averages of i.i.d. variables with finite fourth moments. We divide the $n^{4}$ terms of the expansion for $\left(\sum_{i=1}^{n} d_{i}\right)^{4}$ into four groups, according to the power to which the $d_{i}$ of the greatest $i$ in that term is raised, and then rearrange the sums of the terms in the groups.

So $f_{n}^{4}=\left(\sum d_{i}\right)^{4}=I+I I+I I I+I V$, where

$$
(I)=4 \sum_{i=1}^{n} d_{i}\left(\sum_{j=1}^{i-1} d_{j}\right)^{3},
$$




$$
\begin{aligned}
(I I) & =6 \sum_{i=1}^{n} d_{i}^{2}\left(\sum_{j=1}^{i-1} d_{j}\right)^{2} \\
(I I I) & =4 \sum_{i=1}^{n} d_{i}^{3}\left(\sum_{j=1}^{i-1} d_{j}\right) \\
(I V) & =\sum_{i=1}^{n} d_{i}^{4}
\end{aligned}
$$

Now $\mathrm{E} d_{i}\left(\sum_{j=1}^{i-1} d_{j}\right)^{3}=\mathrm{E}\left[\mathrm{E}\left(d_{i} \mid d_{j}, j<i\right)\left(\sum d_{j}\right)^{3}\right]=\mathrm{E} 0=0$, so $\mathrm{E}(I)=$ 0 . And by $\left.(2.3), \mathrm{E}\left(d_{k}^{2} \mid d_{i}, i<k\right)\right)<C(\gamma)=C$, so $\mathrm{E} d_{i}^{2}\left(\sum_{j=1}^{i-1} d_{j}\right)^{2}=$ $\left.\mathrm{E} \mathrm{E}\left(d_{i}^{2} \mid d_{j}, j<i\right)\left(\sum d_{j}\right)^{2}\right)<C \mathrm{E}\left(\sum d_{j}\right)^{2}<C(i-1)<C n$, and so we get $\mathrm{E}(I I)<C n^{2}$.

Since $\mathrm{E}\left(\left|d_{i}\right|^{3} \mid d_{j} j<i\right)<C(\gamma)=C$ by $(2.3)$ we similarly get $\mathrm{E} d_{i}^{3} \sum_{j=1}^{i-1} d_{j} \leq$ $C \mathrm{E}\left|\sum_{j=1}^{i-1} d_{j}\right| \leq C\left[\mathrm{E}\left(\sum d_{j}\right)^{2}\right]^{1 / 2}=C n^{1 / 2}$, and so $\mathrm{E}(I I I)<C n^{3 / 2}$.

Finally, (2.3) implies $\mathrm{E} d_{i}^{4}<C$, and so $\mathrm{E}(I V)<C n$.

Thus $\mathrm{E} f_{n}^{4}<C n^{2}$, which together with the $p=4$ case of (2.1) and Markov's inequality, gives Lemma 2.1 .

We recall that, to keep our notation brief, all VRJPs on $\{0,1\}$ considered in this section are started at 0 . We put

$$
\xi(t)=\inf \{s: L(s, 0)=t\}
$$

so that under $\mathrm{P}^{a, b}$, we have $\xi(a)=0$.

Lemma 2.2 For all $t \geq a, \mathrm{E}^{a, b} L(\xi(t), 1)=\frac{b}{a} t$.

A proof of this lemma is given via Laplace transforms in the appendix. We now sketch a different proof.

Proof of Lemma 2.2: We will show that $y(t):=\mathrm{E}^{a, b} L(\xi(t), 1)$ satisfies the differential equation $y^{\prime}=y / t$. Since $\mathrm{y}(\mathrm{a})=\mathrm{b}$, this implies Lemma 2.2. If $\Delta$ is a positive number, $L(\xi(t+\Delta), 1)-L(\xi(t), 1)$ is the time spent at 1 while the local time at 0 increases from $t$ to $t+\Delta$. The probability of a 
jump from 0 to 1 in this time interval, given $L(\xi(t), 1)$, is $L(\xi(t), 1) \Delta+o(\Delta)$ as $\Delta \rightarrow 0$, and the duration of the excursion to 1 resulting from this jump has an exponential distribution with rate $t$ between $t$ and $t+\Delta$, so that

$$
\lim _{\Delta \rightarrow 0} \frac{\mathrm{E} L(\xi(t+\Delta), 1)-\mathrm{E} L(\xi(t), 1)}{\Delta}=\frac{\mathrm{E} L(\xi(t), 1)}{t},
$$

and our differential equation is satisfied. It takes a little more work to show that the expectation of the sum of the durations of all the excursions beyond the first is $o(\Delta)$. This argument is omitted.

We put $m_{t}=m_{t}^{a, b}=\frac{L^{a, b}(\xi(t), 1)}{t}$, if $t \geq a$, where the superscript means that we are studying $L(\xi(t), 1) / t$ under $\mathrm{P}^{a, b}$.

Corollary 2.3 The process $m_{t}, t \geq a$, is a martingale.

Proof: This is immediate from Lemma 2.2 and the fact that given $Z_{s}, 0 \leq$ $s \leq \xi(t)$, the process $Z_{y+t}, y \geq 0$, has the same distribution as VRJP on $\{0,1\}$ under $\mathrm{P}^{t, L(\xi(t), 1)}$.

Corollary 2.4 Both the limits $\lim _{t \rightarrow \infty} m_{t}^{a, b}$, and $\lim _{t \rightarrow \infty} \frac{L^{a, b}(t, 1)}{L^{a, b}(t, 0)}$ almost surely exist and are equal and positive.

Proof: Note that the right continuity of the paths of $Z_{t}, t \geq 0$, gives that if $L(t, 0)=s$, then

$$
\frac{L(\xi(s), 1)}{s} \leq \frac{L(t, 1)}{L(t, 0)} \leq \lim _{r \downarrow s} \frac{L(\xi(r), 1)}{s}=\lim _{r \downarrow s} \frac{L(\xi(r), 1)}{r} .
$$

Thus, since $\lim _{t \rightarrow \infty} m_{t}$ exists a.s.,

$$
\lim _{t \rightarrow \infty} \frac{L^{a, b}(t, 1)}{L^{a, b}(t, 0)} \quad \text { exists a.s. }
$$

To complete the proof we will show that the latter limit is strictly positive by showing that

$$
\lim _{t \rightarrow \infty} \frac{L^{a, b}(t, 0)}{L^{a, b}(t, 1)} \quad \text { exists a.s. }
$$


This is done by noting that if $\tau$ is the time of the first jump to 1 , then, conditioned on $\left\{Z_{t}, 0 \leq t \leq \tau\right\}$, the distribution of $1-\mathbf{Z}_{t+\tau}, t \geq 0$, (i.e. we just relabel 0 as 1 and 1 as 0 ), has the distribution of $Z_{t}, t \geq 0$, under $\mathrm{P}^{b, a+\tau}$, so that (2.6) follows from (2.5).

Our treatment of the following lemma parallels that of Lemma 2.2; it is proved in the appendix, and a different proof is sketched in this section.

Lemma 2.5 For all $r \geq a$

$$
\mathrm{E}^{a, b} L(\xi(r), 1)^{2}=-\frac{b}{a}+\frac{a b^{2}+b}{a^{3}} r^{2} .
$$

Proof: We have $L(\xi(r+d r), 1)=L(\xi(r))+\nu \eta$, where $\nu$ is $\operatorname{Bernoulli}(L(\xi(r), 1) d r)$ and $\eta$ is exponential $(r)$, and $\nu$ and $\eta$ are independent given $L(\xi(r), 1)$. Thus, noting $\nu^{2}=\nu$, we have

$$
\begin{aligned}
\mathrm{E} L(\xi(r+d r), 1)^{2} & =\mathrm{E} L(\xi(r), 1)^{2}+2 \mathrm{E}\{L(\xi(r+d r), 1) \mathrm{E}(\nu \mid L(\xi(r), 1))\} \mathrm{E} \eta \\
& +\mathrm{E} \eta^{2} \mathrm{EE}\left(\nu^{2} \mid L(\xi(r), 1)\right) \\
& =\mathrm{E} L(\xi(r), 1)^{2}+\frac{2}{r} \mathrm{E} L(\xi(r), 1)^{2} d r+\frac{2}{r^{2}} \mathrm{E} L(\xi(r), 1) d r \\
& =\mathrm{E} L(\xi(r), 1)^{2}+\frac{2}{r} \mathrm{E} L(\xi(r), 1)^{2} d r+\frac{2 b}{a r} d r,
\end{aligned}
$$

using Lemma 2.2 in the last line.

Thus E $L(\xi(r), 1)^{2}$ satisfies $y^{\prime}=\frac{2}{r} y+\frac{2 b}{a r}$, and $y(a)=b^{2}$, and Lemma 2.5 follows.

Lemma 2.5 immediately gives the $L^{2}$ norm of the martingale $m_{t}^{a, b}, t \geq a$, is finite, since

$$
\mathrm{E}^{a, b}\left(m_{r}\right)^{2}=\frac{a b^{2}+b}{a^{3}}-\frac{b}{a r^{2}}, r \geq a .
$$

The continuous version of $(2.2)$ with $m_{r}$ playing the role of $f_{n}$, along with (2.7) give, putting $m_{\infty}:=\lim _{t \rightarrow \infty} m_{t}$,

$$
\mathrm{E}^{a, b} \sup _{s \geq a}\left(m_{s}-m_{\infty}\right)^{2} \leq 16 \sup _{s \geq a} \mathrm{E}\left(m_{s}-m_{a}\right)^{2}=16 \sup _{s \geq a}\left(\mathrm{E} m_{s}^{2}-m_{a}^{2}\right) \leq 16 \frac{b}{a^{3}},
$$


which, together with (2.4), gives

$$
\mathrm{E}^{a, b} \sup _{t \geq 0}\left(\frac{L(t, 1)}{L(t, 0)}-m_{\infty}\right)^{2} \leq 16 \frac{b}{a^{3}}
$$

Also, we have,

Lemma 2.6 $\mathrm{E}^{a, b} \sup _{y \geq 0}\left|\log \frac{L(y, 1)}{L(y, 0)}\right|^{4}<C(a, b)$.

Proof: Since $(\log x)^{4}<x^{2}, x>1,(2.4)$ together with (2.7) and the continuous version of $(2.1)$ in the case $p=2$ give, if $r^{+}=\max (r, 0)$,

$$
\mathrm{E}^{a, b} \sup _{y \geq 0}\left[\left(\log \frac{L(y, 1)}{L(y, 0)}\right)^{+}\right]^{4} \leq 4 \frac{a b^{2}+b}{a^{3}} .
$$

Let $\tau$ be the time of the first jump of $Z_{t}$ to 1 . Then, given $\tau$, the process $\frac{L(y+\tau, 0)}{L(y+\tau, 1)}, y \geq 0$, has the same distribution as $\frac{L(y, 1)}{L(y, 0)}, y \geq 0$, under $\mathrm{P}^{b+\tau, a}$, and from this and (2.9) it is easy to conclude that

$$
\mathrm{E}^{a, b} \sup _{y \geq 0}\left[\left(\log \frac{L(y, 0)}{L(y, 1)}\right)^{+}\right]^{4}<C(a, b),
$$

since the interval $0 \leq y \leq \tau$ is easily handled. Together (2.9) and (2.10) give Lemma 2.6.

We use the superscript ${ }^{1,1+\exp 1}$ to indicate we are starting VRJP on $\{0,1\}$ with a random initial weight of 1 plus an exponential (1) random variable at one, and 1 at zero, so that the VRJP behaves like VRJP on $\{0,1\}$ with initial weights 1 at both zero and one, started at one, after the first jump to zero. It is easy to conclude from Lemma 2.6 and the concavity of $\log x$ that $\log m_{t}, t \geq 0$, is a supermartingale under $\mathrm{E}^{1,1+\exp 1}$, satisfying

$$
\sup _{t \geq 0} \mathrm{E}^{1,1+\exp 1}\left|\log m_{t}\right|^{4}<\infty .
$$

Furthermore, we will calculate in the appendix the number $\alpha \approx 0.36$, defined by

$$
\alpha=\mathrm{E}^{1,1+\exp 1} \log m_{\infty} .
$$


Now $\mathrm{E}^{1,1+\exp 1} \log m_{t}$ is non-increasing as $t$ increases, and (2.11) implies this convergence is dominated, so that

$$
\mathrm{E}^{1,1+\exp 1} \log m_{t} \downarrow \alpha \text { as } t \rightarrow \infty .
$$

\section{$3 \quad$ VRJP on the nonnegative integers}

In this section, $Y_{t}, t \geq 0$, exclusively stands for VRJP on $\{0,1,2, \ldots\}$ started at 0 , with initial weights all 1 , and we use $L^{Y}(t, k)$ to denote $1+\int_{0}^{t} I\left(Y_{s}=\right.$ $k) d s$, often omitting the superscript. We let $T_{n}=\inf \left\{t: Y_{t}=n\right\}$. We will need the following, which is immediate from the construction of VRJP.

Restriction principle. VRJP observed only at the times when it stays on some subset of consecutive integers $A$, behaves the same way as VRJP restricted to the set $A$. Moreover, it is independent of either the path VRJP to the right of $A$ or the path of VRJP to the left of $A$.

More precisely, let $W_{t}, t \geq 0$, be a VRJP (initial weights are 1 ) on any set of consecutive integers, and let $A$ be a subset of consecutive integers of those integers. Let $T=\inf \left\{t \geq 0: W_{t} \in A\right\}$ be a stopping time, and $k=W_{T} \in A$ be the "port of entry". Put $\delta^{A}(a)=\sup \left\{t: \int_{0}^{t} I\left(W_{s} \in A\right) d s=a\right\}$. Then $H_{a}:=W_{\delta(a)}$ is a VRJP on $A$ started at $k$. If $B$ represents the states to the left or to the right of $A$, then $W_{\delta^{B}(a)}, a \geq 0$, is independent of $W_{\delta^{A}(a)}, a \geq 0$.

Lemma 3.1 If $n>0, \mathrm{P}\left(T_{n}<\infty\right)=1$.

Proof: Since $\sum_{i=0}^{\infty}[L(t, i)-1]=t$, if $\mathrm{P}\left(T_{n}<\infty\right)<1$ there must be a $j$, $0 \leq j<n$, such that $\mathrm{P}(L(\infty, j)=\infty, L(\infty, j+1)<\infty)>0$. Now we use the restriction principle on $\{j, j+1\}$, together with Corollary 2.4, with $j$ relabeled as zero and $j+1$ relabeled as one, to get a contradiction.

Next we observe the following 
Lemma 3.2 Let $1 \leq j<n$. Then given $L\left(T_{n}, i\right), i \geq j+1$, the distribution of $L\left(T_{n}, j\right) / L\left(T_{n}, j+1\right)$ is the distribution of $m_{L\left(T_{n}, j+1\right)}^{1,1+\exp 1}$.

Proof: Note that $L\left(T_{j+1}, j\right)$ has the distribution $1+\exp 1$, while of course $L\left(T_{j+1}, j+1\right) \equiv 1$. The rest of the argument follows from the restriction principle applied to $\{j, j+1\}$, together with the observation that what happens on excursions of $Y_{t}$ to the right of $j+1$ is not influenced by what happens to $Y_{t}$ while it is on $\{0,1,2, \ldots, j+1\}$.

The following proposition establishes the recurrence of VRJP on nonnegative integers.

Proposition 3.3 For all $j \geq 0, L(\infty, j)=\infty$ a.s.

Proof: Suppose that $\mathrm{P}(L(\infty, j)<\infty)>0$ for some $j$. Then $\mathrm{E} 1 / L(\infty, j)>$ 0 . By restriction principle applied to $\{j, j+1, \ldots\}$, and Lemma 3.1, this expectation must be the same for all $j \geq 0$.

We claim that $\mathrm{E}\left(m_{t}^{1,1+\exp 1}\right)^{-1}<1$ for all $t>0$. We know that (see the appendix)

$$
\phi_{a, b}(\lambda)=\mathrm{E} \exp \left(-\lambda m_{t}^{a, b}\right)=e^{b\left(a-\sqrt{\lambda^{2} / t^{2}+2 \lambda+a^{2}}\right)} .
$$

Since $\int_{0}^{\infty} \exp \left(-\lambda m_{t}^{a, b}\right) d \lambda=\frac{1}{m_{t}^{a, b}}$, we have

$$
\begin{aligned}
\mathrm{E} \frac{1}{m_{t}^{1,1+\exp 1}} & =\int_{0}^{\infty} \phi_{1,1+\exp 1}(\lambda) d \lambda \\
& =\int_{0}^{\infty} d \lambda \int_{0}^{\infty} \exp (-u) d u \exp \left((u+1)\left(1-\sqrt{\lambda^{2} / t^{2}+2 \lambda+1}\right)\right) \\
& <\int_{0}^{\infty} d \lambda \int_{0}^{\infty} d u \exp (1-(u+1)(1-\sqrt{2 \lambda+1})) \\
& =\int_{0}^{\infty} \frac{\exp (1-\sqrt{2 \lambda+1})}{\sqrt{2 \lambda+1}} d \lambda=1 .
\end{aligned}
$$

On the other hand, if $\mathrm{P}(L(\infty, j)<\infty)>0$,

$$
\text { E } \quad\left(\frac{1}{L(\infty, j-1)}-\frac{1}{L(\infty, j)}\right)
$$




$$
=\mathrm{E} \quad\left[\mathrm{E}\left(\frac{1}{L(\infty, j-1)}-\frac{1}{L(\infty, j)} \mid L(\infty, j)=t\right)\right]<0,
$$

since by restriction principle, after relabeling $j-1$ as one and $j$ as zero,

$$
\begin{aligned}
\mathrm{E}\left(\frac{1}{L(\infty, j-1)}-\frac{1}{L(\infty, j)} \mid L(\infty, j)=t\right) & =\frac{1}{t} \mathrm{E}^{1,1+\exp 1}\left(\frac{t}{L(\xi(t), 1)}-1\right) \\
& =\frac{1}{t}\left(\mathrm{E} \frac{1}{\left.m_{t}^{1,1+\exp 1}-1\right)<0,}\right.
\end{aligned}
$$

yielding a contradiction.

Now let $R_{i}^{t}=\frac{L(t, i)}{L(t, i+1)}$ and $R_{i}^{n}:=R_{i}^{T_{n}}$. The notational ambiguity will not cause trouble. For $i \geq 0$ put $Z_{i}=\lim _{t \rightarrow \infty} R_{i}^{t}$. Recall that $\alpha$ is defined in (2.12) and calculated in (5.46). Put both $R_{-1}^{t}$ and $Z_{-1}$ equal to 1.

Lemma 3.4 The following hold.

i) $Z_{i}, i \geq 0$, are i.i.d., $\mathrm{E} \log Z_{i}=\alpha$, and the density of $Z_{i}^{-1}$ is the function $f_{\gamma}(x)$ given in the statement of Theorem 1.1 .

ii) $\sum_{k=0}^{\infty} \prod_{i=-1}^{k-1} Z_{i}^{-1}<\infty$ a.s.

iii) $\mathrm{E}\left(\log R_{j}^{n} \mid L\left(T_{n}, i\right), j+1 \leq i \leq n\right)>\alpha, 0 \leq j<n$.

Proof: The first two statements of i), and iii) follow from Lemma 3.2, the definition of $\alpha$, and, in the case of iii), (2.13). For the last statement of i) see formula (5.45) in the appendix. And ii) follows almost immediately from i) and the SLLN, which enables the bounding of the terms of the sum by a geometric series.

Next we state the main result of this section, the almost sure convergence, in $l_{1}$, of the empirical occupational time distribution, Put

$$
p_{k}=\frac{\prod_{i=-1}^{k-1} Z_{i}^{-1}}{\sum_{k=0}^{\infty} \prod_{i=-1}^{k-1} Z_{i}^{-1}} .
$$

Theorem 3.5 The following holds.

$$
\lim _{t \rightarrow \infty} \sum_{k=0}^{\infty}\left|\frac{L(t, k)-1}{t}-p_{k}\right|=0 \text { a.s. }
$$


Before proving Theorem 3.5 we sketch for motivation a short proof of a weaker result. We have

$$
L(t, k)=L(t, 0) \prod_{j=-1}^{k-1}\left(R_{j}^{t}\right)^{-1}=: \Gamma_{k}^{t}, k \geq 0 .
$$

Putting $\Theta_{k}=\prod_{i=-1}^{k-1} Z_{i}^{-1}$, the definition of $Z_{i}$ gives

$$
\lim _{t \rightarrow \infty} \frac{\Gamma_{k}^{t}}{\Gamma_{k+1}^{t}}=\frac{\Theta_{k}}{\Theta_{k+1}} \text { a.s., } k \geq 0 .
$$

Now if $n$ is fixed and $a_{k}^{t}, 0 \leq k \leq n, t \geq 0$, and $b_{k}, 0 \leq k \leq n$ are positive numbers such that

$$
\lim _{t \rightarrow \infty} \frac{a_{k}^{t}}{a_{k+1}^{t}}=\frac{b_{k}}{b_{k+1}}
$$

then

$$
\lim _{t \rightarrow \infty} \frac{a_{k}^{t}}{\sum_{i=0}^{n} a_{i}^{t}}=\frac{b_{k}}{\sum_{i=0}^{n} b_{i}}, 0 \leq k \leq n
$$

which shows

$$
\lim _{t \rightarrow \infty} \frac{L(k, t)}{\sum_{i=0}^{n} L(i, t)}=\frac{\Theta_{k}}{\sum_{i=0}^{n} \Theta_{i}} .
$$

The last equality together with Proposition 3.3 implies that if $0 \leq k \leq n$

$$
\lim _{t \rightarrow \infty} \frac{L(k, t)-1}{\sum_{i=0}^{n}(L(i, t)-1)}=\frac{\Theta_{k}}{\sum_{i=0}^{n} \Theta_{i}},
$$

a junior version of Theorem 3.5 , since $\sum_{i=0}^{\infty}(L(i, t)-1)=t$.

The following lemma is a more precise version of the simple fact about sequences just used.

Lemma 3.6 Let $a_{i}$ and $b_{i}, 0 \leq i \leq n$, be positive numbers, and let $\varepsilon>0$. Put $A=\sum_{i=0}^{n} a_{i}$ and $B=\sum_{i=0}^{n} b_{i}$. Then

$$
\sum_{i=0}^{n} \frac{\left|a_{i}-b_{i}\right|}{a_{i}}<\varepsilon \text { implies } \sum_{i=0}^{n}\left|\frac{a_{i}}{A}-\frac{b_{i}}{B}\right|<\frac{2 \varepsilon}{1-\varepsilon} .
$$


Proof: The hypotheses imply $|A-B|<\varepsilon A$, and so

$$
\begin{aligned}
\sum_{i=0}^{n}\left|\frac{a_{i}}{A}-\frac{b_{i}}{B}\right| & =\sum_{i=0}^{n}\left|\frac{a_{i}(B-A)+\left(a_{i}-b_{i}\right) A}{A B}\right| \leq \frac{|B-A|}{B}+\frac{\sum\left|b_{i}-a_{i}\right|}{B} \\
& <\frac{\varepsilon}{1-\varepsilon}+\frac{\varepsilon}{1-\varepsilon} .
\end{aligned}
$$

Now since $L\left(T_{n}, n\right)=1$, we have, recalling $R_{n-i}^{T_{n}}$ is shortened to $R_{n-i}^{n}$, that $\log L\left(T_{n}, n-k\right)=\sum_{i=0}^{k} \log R_{n-i}^{n}$. Put $\Delta_{i}^{n}=\mathrm{E}\left(\log R_{n-i}^{n} \mid R_{n-j}^{n}, 0 \leq\right.$ $j<i), 1 \leq i \leq n$, and $D_{i}^{n}=\log R_{n-i}^{n}-\Delta_{i}^{n}$. (Sometimes we drop the superscript.) Then Lemma 3.4 iii) implies

$$
\Delta_{i}^{n} \geq \alpha \text { a.s., } 1 \leq i \leq n
$$

and of course $D_{i}^{n}, 1 \leq i \leq n$, is a martingale difference sequence. Furthermore, Lemma 3.2 and (2.11) imply $\mathrm{E}\left(\left|\log R_{n-i}^{n}\right|^{4} \mid R_{n-j}^{n}, 1 \leq j<i\right)<C$, $1 \leq i \leq n$, which in turn implies

$$
\mathrm{E}\left(D_{i}^{4} \mid R_{n-j}^{n}, 1 \leq j<i\right)<C, 1 \leq i \leq n,
$$

where the $C$ is absolute, especially it does not depend on $i$ or $n$.

Thus for any $\varepsilon>0$, according to Lemma 2.1,

$$
\mathrm{P}\left(\left|\sum_{i=1}^{n} D_{i}\right|>\varepsilon n\right)<\frac{C(\varepsilon)}{n^{2}},
$$

which with (3.14) implies $\mathrm{P}\left(\sum_{i=1}^{n} D_{i}+\Delta_{i}<(\alpha-\varepsilon) n\right)<C(\varepsilon) / n^{2}$, or equivalently,

$$
\mathrm{P}\left(\log L\left(T_{n}, 0\right)<(\alpha-\varepsilon) n\right)<\frac{C(\varepsilon)}{n^{2}} .
$$

This inequality, Borel-Cantelli, and the fact that $L\left(T_{n}, 0\right)-1<T_{n}$, imply

$$
\mathrm{P}\left(\liminf _{n \rightarrow \infty} \log T_{n} / n \geq \alpha\right)=1
$$


or equivalently

$$
\limsup _{t \rightarrow \infty} \frac{\max _{0 \leq s \leq t} X_{s}}{\log t} \leq \alpha^{-1} \text { a.s. }
$$

In the following, if $a<b$ are not necessarily integers, we use $\sum_{i=a}^{b} r_{i}$ to designate the sum of those $r_{i}$ for all $i$ satisfying $a \leq i \leq b$. We let $\theta$ be a fixed number between 0 and 1 satisfying $\theta \log 2<\frac{1}{4}$, which guarantees

$$
\frac{2^{(n+1) \theta}}{e^{n / 4}}<2 \beta^{n} \text { where } \beta:=e^{\theta \log 2-\frac{1}{4}}<1 .
$$

The proof of Theorem 3.5 will be completed by establishing the following two limits. We have

$$
\sup _{t \geq T_{n}} \sum_{i=0}^{n(1-\theta)}\left|\frac{L(t, i)-1}{\sum_{j=0}^{n(1-\theta)}(L(t, j)-1)}-p_{i}\right| \rightarrow 0 \text { as } n \rightarrow \infty
$$

and

$$
\sum_{i=n(1-\theta)}^{n+1} \frac{L\left(T_{n+1}, i\right)}{e^{n / 4}} \rightarrow 0 \text { as } n \rightarrow \infty
$$

To see that (3.20) and (3.21) imply Theorem 3.5, observe that to prove Theorem 3.5, it suffices to prove

$$
\sup _{T_{n} \leq t \leq T_{n+1}} \sum_{k=0}^{n+1}\left|\frac{L(t, k)-1}{\sum_{j=0}^{n+1}(L(t, j)-1)}-p_{k}\right| \rightarrow 0 \text { as } n \rightarrow \infty
$$

since $L\left(T_{n+1}, k\right)=1$ if $k>n+1$, and $\sum_{k=0}^{\infty} p_{k}=1$. Now (3.20) obviously implies

$$
\sup _{T_{n} \leq t \leq T_{n+1}} \sum_{k=0}^{n(1-\theta)}\left|\frac{L(t, k)-1}{\sum_{j=0}^{n(1-\theta)}(L(t, j)-1)}-p_{k}\right| \rightarrow 0 \text { as } n \rightarrow \infty .
$$

Furthermore, if $n \geq 1$,

$$
\sup _{T_{n} \leq t \leq T_{n+1}} \sum_{k=n(1-\theta)}^{n+1}\left|\frac{L(t, k)-1}{\sum_{j=0}^{n+1}(L(t, j)-1)}-p_{k}\right|
$$




$$
\begin{aligned}
& \leq \sup _{T_{n} \leq t \leq T_{n+1}} \sum_{k=n(1-\theta)}^{n+1}\left|\frac{L(t, k)-1}{\sum_{j=0}^{n+1}(L(t, j)-1)}\right|+\sum_{k=n(1-\theta)}^{n+1} p_{k} \\
& \leq \frac{\sum_{k=n(1-\theta)}^{n+1} L\left(T_{n}, k\right)}{T_{n}}+\sum_{k=n(1-\theta)}^{\infty} p_{k} .
\end{aligned}
$$

The second sum here clearly approaches 0 as $n \rightarrow \infty$, and since $T_{n} \geq e^{n / 4}$ for large enough $n$, by (3.17) and (5.46), (3.21) gives that the first does also.

We first prove (3.21), then (3.20). Using Lemma 3.2 and Corollary 2.3 we have, for $0 \leq j \leq n$,

$$
\begin{aligned}
\mathrm{E} L\left(T_{n}, j\right) & =\mathrm{EE}\left(L\left(T_{n}, j\right) \mid L\left(T_{n}, j+1\right)\right) \\
& =\mathrm{E} L_{n}\left(T_{n}, j+1\right) \mathrm{E}\left(\frac{L\left(T_{n}, j\right)}{L\left(T_{n}, j+1\right)} \mid L\left(T_{n}, j+1\right)\right) \\
& =\mathrm{E} L\left(T_{n}, j+1\right) \mathrm{E}^{1,1+\exp 1} m_{L\left(T_{n}, j+1\right)} \\
& =\mathrm{E} L\left(T_{n}, j+1\right) \mathrm{E}^{1,1+\exp 1} m_{1}=2 \mathrm{E} L\left(T_{n}, j+1\right) .
\end{aligned}
$$

Together with $\mathrm{E} L\left(T_{n}, n\right)=1$ this gives $\mathrm{E} L\left(T_{n}, k\right)=2^{n-k}, 0 \leq k \leq n$.

Thus

$$
\sum_{n=1}^{\infty} \sum_{k=n(1-\theta)}^{n+1} \frac{\mathrm{E} L\left(k, T_{n+1}\right)}{e^{n / 4}}<\infty,
$$

using (3.19), and (3.21) follows.

Next we prove (3.20). We observe

$$
\begin{aligned}
& \sup _{t \geq T_{n}} \sum_{i=0}^{n(1-\theta)}\left|\frac{L(t, i)-1}{\sum_{j=0}^{n(1-\theta)}(L(t, j)-1)}-\frac{L(t, i)}{\sum_{j=0}^{n(1-\theta)} L(t, j)}\right| \\
& \leq \sup _{t \geq T_{n}} \frac{n(1-\theta)+2}{\sum_{j=0}^{n(1-\theta)}(L(t, j)-1)},
\end{aligned}
$$

which follows immediately by putting the difference of the quotients on the LHS of (3.22) over a common denominator. Since $\sum_{j=0}^{n(1-\theta)} L(t, j)-1 \geq$ $T_{\lceil n(1-\theta)\rceil}$, if $t \geq T_{n}$, where $\lceil\cdot\rceil$ is the greatest integer function, (3.17) shows that that suprema to the right of the inequality in (3.22) approaches 0 as 
$n \rightarrow \infty$, and thus the suprema to the left does. This implies that the following inequality is equivalent to (3.20).

$$
\sup _{t \geq T_{n}} \sum_{i=0}^{n(1-\theta)}\left|\frac{L(t, i)}{\sum_{j=0}^{n(1-\theta)} L(t, j)}-p_{i}\right| \rightarrow 0 \text { as } n \rightarrow \infty .
$$

To prove (3.23), we rewrite it as

$$
\sup _{t \geq T_{n}} \sum_{k=0}^{n(1-\theta)}\left|\frac{\prod_{i=-1}^{k-1}\left(R_{i}^{t}\right)^{-1}}{\sum_{j=0}^{n(1-\theta)} \prod_{i=-1}^{j-1}\left(R_{i}^{t}\right)^{-1}}-\frac{\prod_{i=-1}^{k-1} Z_{i}^{-1}}{\sum_{j=0}^{n(1-\theta)} \prod_{i=-1}^{j-1} Z_{i}^{-1}}\right| \rightarrow 0
$$

as $n \rightarrow \infty$, and we note that using Lemma 3.6 , to prove (3.23) it suffices to prove

$$
\sup _{t \geq T_{n}} \sum_{k=0}^{n(1-\theta)}\left|\frac{\prod_{i=-1}^{k-1}\left(R_{i}^{t}\right)^{-1}-\prod_{i=-1}^{k-1} Z_{i}^{-1}}{\prod_{i=-1}^{k-1} Z_{i}^{-1}}\right| \rightarrow 0 \text { as } n \rightarrow \infty
$$

which reduces to

$$
\sup _{t \geq T_{n}} \sum_{k=0}^{n(1-\theta)}\left|1-\prod_{i=-1}^{k-1}\left(1+\frac{Z_{i}-R_{i}^{t}}{R_{i}^{t}}\right)\right| \rightarrow 0 \text { as } n \rightarrow \infty .
$$

Now $\left|1-\prod_{i=0}^{m}\left(1+a_{i}\right)\right|<\exp \left(\sum_{i=0}^{m}\left|a_{i}\right|\right)-1 \leq 2 \Sigma\left|a_{i}\right|$ if $\Sigma\left|a_{i}\right|<0.1$, and so (3.24) follows from

$$
n \cdot \sup _{t \geq T_{n}} \sum_{k=0}^{n(1-\theta)}\left|\frac{Z_{i}-R_{i}^{t}}{R_{i}^{t}}\right| \rightarrow 0 \text { as } n \rightarrow \infty,
$$

The initial $n$ in (3.25) is an upper bound (if $n$ is large) for the number of summands $k$ in $(3.24), k=0,1, \ldots, n(1-\theta)$. Now exactly as we proved (3.16), we have

$$
\mathrm{P}\left(\sum_{i=1}^{\theta n-1} D_{i}+\Delta_{i}<0.2 \theta n \alpha\right)<\frac{C}{n^{2}}
$$

Also, Lemma 2.1 and (3.14), or even the weaker version of (3.14) with $\alpha$ replaced by zero, imply

$$
\mathrm{P}\left(\inf _{\theta n \leq k \leq n} \sum_{i=\theta n}^{k} D_{i}+\Delta_{i}<-0.1 \theta(1-\theta) n \alpha\right)<\frac{C}{n^{2}} .
$$


Together (3.26) and (3.27) give

$$
\mathrm{P}\left(\inf _{\theta n-1 \leq k \leq n} \sum_{i=0}^{k} D_{i}+\Delta_{i}<0.1 \theta n \alpha\right)<\frac{C}{n^{2}},
$$

or, equivalently,

$$
\mathrm{P}\left(L\left(T_{n}, i\right) \geq e^{0.1 \theta n}, 0 \leq i \leq n(1-\theta)+1\right)>1-\frac{C}{n^{2}} .
$$

Let $G_{i}=G_{i}^{n}=\left\{L\left(T_{n}, i\right)>e^{0.1 \theta n}\right\}$. Then (3.28) may be restated as

$$
\mathrm{P}\left(\bigcup_{i=0}^{n(1-\theta)+1} G_{i}^{c}\right)<\frac{C}{n^{2}},
$$

where the superscript ${ }^{c}$ denotes complement.

Now conditioned on $L\left(T_{n}, i\right)$ and $L\left(T_{n}, i+1\right)$, the distribution of $Y_{t}$, $t \geq T_{n}$, restricted to $\{i, i+1\}$ has the distribution of the two state walk of Section $2, Z_{t}, t \geq 0$, under $\mathrm{P}^{L\left(T_{n}, i+1\right), L\left(T_{n}, i\right)}$ if we relabel $i+1$ as 0 and $i$ as 1. Thus (2.8) implies

$$
\begin{aligned}
& \mathrm{E}_{\sup _{t \geq T_{n}}\left(Z_{i}-R_{i}^{t}\right)^{2} I\left(G_{i+1}\right)} \\
= & \mathrm{EE}\left(\sup _{t \geq T_{n}}\left(Z_{i}-R_{i}^{t}\right)^{2} \mid L\left(T_{n}, i+1\right), L\left(T_{n}, i\right)\right) I\left(G_{i+1}\right) \\
\leq & \mathrm{E} \frac{16 L\left(T_{n}, i\right)}{L\left(T_{n}, i+1\right)^{3}} I\left(G_{i+1}\right) \leq 16\left(e^{-0.1 \theta n}\right)^{2} \mathrm{E} R_{i}^{n}=32 e^{-0.2 \theta n},
\end{aligned}
$$

using Corollary 2.3 and the fact that $R_{i}^{n}$ has the distribution of $m_{L\left(T_{n}, i+1\right)}$ under $\mathrm{P}^{1,1+\exp 1}$, so $\mathrm{E} R_{i}^{n}=\mathrm{E}^{1,1+\exp 1} m_{0}=2$.

Thus,

$$
\begin{aligned}
& \mathrm{E}_{\sup _{t \geq T_{n}}}\left|\frac{Z_{i}-R_{i}^{t}}{R_{i}^{t}}\right| I\left(G_{i+1}\right) \leq\left[\mathrm{E}_{\sup _{t \geq T_{n}}}\left(Z_{i}-R_{i}^{t}\right)^{2} I\left(G_{i+1}\right)\right]^{\frac{1}{2}} \\
& \times \quad\left[\mathrm{E} \sup _{t \geq 0} \frac{1}{\left(R_{i}^{t}\right)^{2}}\right]^{\frac{1}{2}} \leq C e^{-0.1 \theta n},
\end{aligned}
$$

using (3.30). That $\mathrm{E} \sup _{t \geq T_{n}}\left(R_{i}^{t}\right)^{-2}$ is finite follows from the restriction principle and the fact that $\sup _{t \geq 0}\left(R_{i}^{t}\right)^{-1}$ has the same distribution as $\sup _{s \geq 1} m_{s}$ 
under $P^{1,1}$, using the continuous version of Lemma 2.1 and the sentence which includes (2.7). Finally, to complete the proof of (3.25) and thus (3.20), we have, for large enough $\mathrm{n}$,

$$
\begin{aligned}
& \left\{n \sup _{t \geq T_{n}} \sum_{i=0}^{n(1-\theta)}\left|\frac{Z_{i}-R_{i}^{t}}{R_{i}^{t}}\right| \geq e^{-0.1 \theta n}\right\} \subset\left(\bigcup_{i=0}^{n(1-\theta)} G_{i}^{c}\right) \\
\cup & \bigcup_{i=0}^{n(1-\theta)}\left\{\sup _{t \geq T_{n}}\left|\frac{Z_{i}-R_{i}^{t}}{R_{i}^{t}}\right| I\left(G_{i+1}\right)>n^{-1} e^{-0.05 \theta n}\right\}
\end{aligned}
$$

And

$$
\begin{aligned}
& \mathrm{P}\left(\bigcup_{i=0}^{n(1-\theta)}\left\{\sup _{t \geq T_{n}}\left|\frac{Z_{i}-R_{i}^{t}}{R_{i}^{t}}\right| I\left(G_{i+1}\right)>n^{-1} e^{-0.05 \theta n}\right\}\right) \\
\leq & \sum_{i=0}^{n(1-\theta)} \mathrm{P}\left(\sup _{t \geq T_{n}}\left|\frac{Z_{i}-R_{i}^{t}}{R_{i}^{t}}\right| I\left(G_{i+1}\right)>n^{-1} e^{-0.05 \theta n}\right) \\
\leq & \sum_{i=0}^{n(1-\theta)} n e^{0.05 \theta n} \mathrm{E} \sup _{t \geq T_{n}}\left|\frac{Z_{i}-R_{i}^{t}}{R_{i}^{t}}\right| I\left(G_{i+1}\right) \leq C n^{2} e^{-0.05 \theta n}<\frac{C}{n^{2}},
\end{aligned}
$$

using (3.31). And this inequality, together with (3.29) and (3.32) and BorelCantelli, establish (3.25).

The next theorem is a one-sided version of Theorem 1.2.

Theorem 3.7 The following holds.

$$
\lim _{t \rightarrow \infty} \frac{\max _{0 \leq s \leq t} X_{s}}{\log t}=\alpha^{-1} \text { a.s. }
$$

Proof: We will show that given $\varepsilon>0$, there is a constant $C(\varepsilon)$ such that

$$
P\left(T_{n}>e^{n(\alpha+\varepsilon)}\right)<\frac{C(\varepsilon)}{n^{2}} n \geq 1 .
$$

This inequality together with Borel-Cantelli shows $\lim \sup _{n \rightarrow \infty} \log T_{n} / n \leq$ $\alpha$, which implies

$$
\liminf _{t \rightarrow \infty} \frac{\max _{0 \leq s \leq t} X_{s}}{\log t} \geq \alpha^{-1}
$$


and which, with (3.18), gives (3.33).

We know $\mathrm{E}^{1,1+\exp 1} \log m_{t}$ decreases to $\alpha$. Let $K=\mathrm{E}^{1,1+\exp 1} \log m_{1}$. Let $\gamma>0$, and let the integer $N$ satisfy

$$
\mathrm{E}^{1,1+\exp 1} \log m_{e^{0.1 \theta N}}<(1+\gamma) \alpha
$$

Here and below we use the notation of the proof of Theorem 3.5.

Now (3.35) implies $\Delta_{i} I\left(G_{i+1}\right)<(1+\gamma) \alpha, 0 \leq i \leq n$, and so on the intersection of the $G_{i}, 1 \leq i \leq n$,

$$
\sum_{i=1}^{n} \Delta_{i}=\sum_{i=1}^{\theta n} \Delta_{i}+\sum_{i=\theta n+1}^{n} \Delta_{i} \leq K \theta n+\sum_{i=\theta n+1}^{n} \Delta_{i}
$$

Thus if $n \geq N$,

$$
\begin{aligned}
\sum_{i=1}^{n} \Delta_{i} I\left(\bigcap_{i=1}^{n(1-\theta)+1} G_{i}\right) \leq & K \theta n+[(1-\theta) n+1](1+\gamma) \alpha \\
=: & n f(\theta, \gamma),
\end{aligned}
$$

where $f(\theta, \gamma)=K \theta+(1-\theta)(1+\gamma) \alpha+\Theta(1 / n)$.

Lemma 2.1 gives

$$
\mathrm{P}\left(\sup _{1 \leq k \leq n} \sum_{i=1}^{k} D_{i}>\gamma n\right)<\frac{C(\gamma)}{n^{2}}
$$

and so

$$
\mathrm{P}\left(\sup _{1 \leq k \leq n} \sum_{i=1}^{k} D_{i}+\Delta_{i}>n f(\theta, \gamma)+n \gamma, \bigcap_{i=1}^{n(1-\theta)+1} G_{i}\right)<\frac{C(\gamma)}{n^{2}}
$$

when $n \geq N$. Together with (3.29), this gives that if $n \geq N$,

$$
\mathrm{P}\left(\sum_{i=1}^{k} D_{i}+\Delta_{i}<n f(\theta, \gamma)+n \gamma, 1 \leq k \leq n\right)>1-\frac{C}{n^{2}},
$$

so that

$$
\mathrm{P}\left(\log L\left(T_{n}, i\right)<n f(\theta, \gamma)+n \gamma, 0 \leq i \leq n\right)>1-\frac{C}{n^{2}} .
$$


Since $\sum_{i=0}^{n-1}\left(L\left(T_{n}, i\right)-1\right)=T_{n}$, we get

$$
\mathrm{P}\left(T_{n}<C_{1} n e^{n[f(\theta, \gamma)+\gamma]}\right)>1-\frac{C}{n^{2}}, n \geq N
$$

Now if we choose $\theta$ and $\gamma$ so small that $f(\theta, \gamma)+\gamma<\alpha+\varepsilon$, this implies (3.34).

\section{VRJP on the integers.}

We begin this section by describing the random variables $V_{i}$ of Theorem 1.1. Then we prove Theorem 1.1 and use it and Theorem 3.7 to prove Theorem 1.2. Let $X=X_{t}, t \geq 0$, be a VRJP on the integers started at 0 . Let $X_{s}^{+}, s \geq 0$, be $X$ restricted to the nonnegative integers, and let $X_{s}^{-}, s \geq 0$, be $X$ restricted to the non-positive integers. Then both $X^{+}$and $\left(-X^{-}\right)$ are VRJP's on the nonnegative integers. Let $Z_{i}^{+}, i>0$ be the variables defined for $X^{+}$exactly as the variables $Z_{i}$ were defined for $Y_{t}, t \geq 0$, in Section 3 , and let $Z_{i}^{-}$be the analogous variables for $X^{-}$. Then by Theorem 3.5 $\left\{Z_{i}^{+}, 1 \leq i<\infty, Z_{i}^{-}, 1 \leq i<\infty\right\}$ are i.i.d. random variables, each having the density function given in the statement of Theorem 1.1, as shown in the appendix.

Put

$$
W_{k}= \begin{cases}\prod_{i=1}^{k}\left(Z_{i}^{+}\right)^{-1}, & k>0, \\ 1, & k=0, \\ \prod_{i=1}^{k}\left(Z_{i}^{-}\right)^{-1}, & k<0,\end{cases}
$$

and put $W=\sum_{i=-\infty}^{\infty} W_{i}$, and $V_{k}=W_{i} / W$. We now prove Theorem 1.1, with $V_{i}$ as just constructed.

Let $\delta(t)=\int_{0}^{t} I\left(X_{s}=0\right) d s, \eta(t)=\int_{0}^{t} I\left(X_{s}>0\right) d s$, and $\mu(t)=\int_{0}^{t} I\left(X_{s}<\right.$ 0) $d s$. Then

$$
\delta(t)+\eta(t)+\mu(t)=t
$$


and using the restriction principle we get both

$$
\lim _{t \rightarrow \infty} \frac{\int_{0}^{t} I\left(X_{s}=j\right) d s}{\eta(t)+\delta(t)}=\frac{W_{j}}{\sum_{i=0}^{\infty} W_{i}}, j \geq 0
$$

and

$$
\lim _{t \rightarrow \infty} \frac{\int_{0}^{t} I\left(X_{s}=j\right) d s}{\mu(t)+\delta(t)}=\frac{W_{j}}{\sum_{i=0}^{\infty} W_{-i}}, j \leq 0
$$

Let $\bar{\delta}(t), \bar{\eta}(t)$, and $\bar{\mu}(t)$ stand for $\delta(t) / t, \eta(t) / t$, and $\mu(t) / t$ respectively. Then (4.37) and the versions of (4.38) and (4.39) for $j=0$ give the following three equations:

$$
\begin{aligned}
\bar{\delta}(t)+\bar{\eta}(t)+\bar{\mu}(t) & =1 \\
\lim _{t \rightarrow \infty} \frac{\bar{\delta}(t)}{\delta(t)+\bar{\eta}(t)} & =\frac{W_{0}}{\sum_{i=0}^{\infty} W_{i}} \\
\lim _{t \rightarrow \infty} \frac{\bar{\delta}(t)}{\delta(t)+\bar{\mu}(t)} & =\frac{W_{0}}{\sum_{i=0}^{\infty} W_{-i}}
\end{aligned}
$$

Thus

$$
\begin{aligned}
\lim _{t \rightarrow \infty} \bar{\delta}(t) & =W_{0} / W \\
\lim _{t \rightarrow \infty} \bar{\eta}(t) & =\sum_{i=1}^{\infty} W_{i} / W \\
\lim _{t \rightarrow \infty} \bar{\mu}(t) & =\sum_{i=1}^{\infty} W_{-i} / W .
\end{aligned}
$$

The equations (4.38), (4.39), and (4.41) imply Theorem 1.1.

Proof of Theorem 1.2: From (3.33) and the restriction principle we have

$$
\lim _{t \rightarrow \infty} \frac{\max _{0 \leq s \leq t} X_{s}}{\log [\delta(t)+\eta(t)]}=\alpha^{-1} \text { a.s. }
$$

Equations (4.41) together with (4.42) prove Theorem 1.2. 


\section{$5 \quad$ Appendix}

To describe the distribution of $L(\xi(t), 1)$, defined in Section 2 immediately before Lemma 2.2 , we will calculate its Laplace transform $\phi^{a, b}(\lambda, t)=$

$\mathrm{E}^{a, b} e^{-\lambda L(\xi(t), 1)}, \lambda \geq 0$. Further we will omit the superscript ${ }^{a, b}$ unless it makes our arguments ambiguous.

Denote $w(t):=L(\xi(t), 1)$ and observe that

$$
w(t+d t)=w(t)+\nu \eta
$$

where $\nu$ is a Bernoulli $(w(t) d t)$ random variable and $\eta$ is an exponential $(t)$ random variable, which, given $w$ and $t$, are independent of anything. Hence

$$
\phi(\lambda, t+d t)=\mathrm{E}\left(e^{-\lambda w-\lambda \eta \nu}\right)=\mathrm{E}\left[e^{-\lambda w} \mathrm{E}\left(e^{-\lambda \eta \nu} \mid w\right)\right]
$$

The inner conditional expectation is easy to compute:

$$
\begin{aligned}
\mathrm{E}\left(e^{-\lambda \eta \nu} \mid w\right) & =(1-w d t) \times 1+w d t \times \mathrm{E}\left(e^{-\lambda \eta}\right) \\
& =(1-w d t)+w d t \times \frac{t}{\lambda+t}=1-w d t \frac{\lambda}{\lambda+t}
\end{aligned}
$$

Plugging this into (5.43) yields

$$
\phi(\lambda, t+d t)-\phi(\lambda, t)=-\frac{\lambda}{\lambda+t} \mathrm{E}\left(w e^{-\lambda w}\right) d t
$$

whence, since $\mathrm{E}\left(w(t) e^{-\lambda w(t)} \mid t\right)=-\partial \phi(\lambda, t) / \partial \lambda$,

$$
\frac{\partial \phi}{\partial t}=\frac{\lambda}{\lambda+t} \cdot \frac{\partial \phi}{\partial \lambda} .
$$

The natural boundary conditions are

$$
\begin{aligned}
\phi(\lambda, a) & =e^{-\lambda b}, \\
\phi(0, \cdot) & =1 .
\end{aligned}
$$

Solving this (see Section 5.1) we obtain

$$
\phi^{a, b}(\lambda, t)=e^{b\left(a-\sqrt{\lambda^{2}+2 \lambda t+a^{2}}\right)} .
$$


Though we are not able to invert Laplace transform (5.44) for every $t$, it still gives us Lemmas 2.2 and 2.5

$$
\begin{aligned}
\mathrm{E}^{a, b} w(t) & =\frac{b}{a} \cdot t, \\
\mathrm{E}^{a, b}[w(t)]^{2} & =\frac{b\left(t^{2}-a^{2}+t^{2} a b\right)}{a^{3}}
\end{aligned}
$$

by differentiating $\phi^{a, b}(\lambda, t)$ once and twice at $\lambda=0$.

Next, we want to calculate explicitly the distribution of $\gamma:=m_{\infty}^{1,1}=$ $\lim _{t \rightarrow \infty} \frac{w(t)}{t}$ which exists by Corollary 2.4. By interchanging the integration and the limit we obtain

$$
\mathrm{E}^{1,1} e^{-\lambda \gamma}=\lim _{t \rightarrow \infty} \phi^{1,1}(\lambda / t, t)=e^{1-\sqrt{1+2 \lambda}}
$$

Using Laplace transform, we can, for example, compute moments of $\gamma$ :

$$
\mathrm{E} \gamma=1, \quad \mathrm{E} \gamma^{2}=2, \quad \mathrm{E} \gamma^{3}=7, \quad \mathrm{E} \gamma^{4}=37, \quad \mathrm{E} \gamma^{5}=266, \ldots
$$

The inversion of $\mathrm{E}^{1,1} e^{-\lambda \gamma}$ requires an integration on a complex plane. We omit these calculations, presenting only the result. The density of the distribution of $\gamma=m_{\infty}^{1,1}$ for $x>0$ is

$$
f_{\gamma}(x)=\frac{1}{2 \pi} \int_{-\infty}^{+\infty} e^{1-\sqrt{-2 i \lambda+1}} e^{-i \lambda x} d \lambda=\frac{e^{1-\frac{1}{2}\left(x+x^{-1}\right)}}{\sqrt{2 \pi x^{3}}}
$$

(one can quite easily verify that its Laplace transform coincides with $e^{1-\sqrt{1+2 \lambda}}$ ). This density is also the density of $m_{\infty}^{1,1+\exp 1}$, and is the density $f_{\gamma}$ of Theorem 1.1. Moreover, we can present the formula for c.d.f. of $\gamma$ :

$$
F_{\gamma}(x)=1-\Phi\left(\frac{1}{\sqrt{x}}-\sqrt{x}\right)+e^{2}\left[1-\Phi\left(\frac{1}{\sqrt{x}}+\sqrt{x}\right)\right], x>0
$$

where $\Phi(\cdot)$ is a c.d.f. of a normal zero-one distribution.

To calculate $\alpha$ in (2.12) observe that $m_{\infty}^{1,1+\exp 1}$ has the same distribution as $1 / m_{\infty}^{1,1}=1 / \gamma$. Consequently,

$$
\alpha=\int_{0}^{\infty} \log (x) f_{1 / \gamma} d x=\int_{0}^{\infty} \log x \cdot \frac{\exp \left(1-\frac{x}{2}-\frac{1}{2 x}\right)}{\sqrt{2 \pi x}} d x=0.3613 \ldots
$$




\subsection{Solution of the equation $(-x) \phi_{x}^{\prime}+(x+y) \phi_{y}^{\prime}=0$}

This equation is a linear PDE to which we can apply a standard technique. We will look for a solution in the area where $x \geq 0$ and $y \geq 1$ with a boundary condition

$$
\phi(x, a)=e^{-b x}
$$

Let $\mathbf{v}(x, y)=(-y, x+y)$ be a column vector, then the equation is equivalent to

$$
\mathbf{v} \cdot \nabla \phi=0
$$

where $\cdot$ denotes a scalar product and $\nabla \phi$ is a gradient of $\phi$. Thus, $\phi(x, y)$ must be constant along the solutions of the equation $\dot{\mathbf{z}}=\mathbf{v}$, where $\mathbf{z}=(x, y)$. Solving the system

$$
\left\{\begin{array}{l}
\dot{x}=-x \\
\dot{y}=x+y
\end{array}\right.
$$

we obtain $x(t)=C_{1} e^{-t}, y(t)=C_{2} e^{t}-\frac{1}{2} C_{1} e^{-t}$. Hence, $2 C_{1} C_{2}=x(x+2 y)$, and any solution $\phi(x, y)$ to the PDE must be a function of one argument $x(x+2 y)$.

If this curve $(x(t), y(t))$ intersects the horizontal line $y=a$ at point $\tilde{x} \geq 0$, then $x(x+2 y)=\tilde{x}(\tilde{x}+2 a)$ and $\tilde{x}=-a+\sqrt{x(x+2 y)+a^{2}}$ (we took a positive sign at the square root since $\tilde{x}$ must be non-negative). On the other hand, $\phi(\tilde{x}, a)=e^{-\tilde{x} b}$, therefore

$$
\phi(x, y)=\phi(\tilde{x}, a)=e^{b\left(a-\sqrt{x(x+2 y)+a^{2}}\right)} .
$$

\section{References}

[1] Benaim, M., Ledoux, M., and Raimond, O. (2000) Self interacting diffusions. Preprint. 
[2] Coppersmith, D. and Diaconis, P. (1987). Random walks with reinforcement. Unpublished manuscript.

[3] Davis, B. (1990). Reinforced random walk. Prob. Th. Rel. Fields 84, pp. $203-229$.

[4] Davis, B. (1999). Reinforced and perturbed random walks. Random walks, Bolyai Soc. Math. Stud., 9, Budapest, pp. 113 - 126.

[5] Doob, J.L. (1953) Stochastic processes. John Wiley and Sons, New York.

[6] Pemantle, R. (1988). Phase transition in reinforced random walk and RWRE on trees. Ann. Probab. 16, pp. 1229 - 1241.

[7] Pemantle, R. and Volkov, S. (1999). Vertex-reinforced random walk on Z has finite range. Ann. Probab. 27, pp. $1368-1388$.

[8] Sellke, T. (1994) Reinforced Random Walk on the d-dimensional Integer Lattice, Technical Report \#94-26, Department of Statistics, Purdue University.

[9] Tóth, B. (1999) Self-interacting random motions - a survey. Random walks, Bolyai Soc. Math. Stud., 9, Budapest, pp. $349-384$.

[10] Volkov, S. (2000) Vertex-Reinforced Random Walk on Arbitrary Graphs Ann, Probab. to appear. 